\title{
Parameter Estimation Based on the Frèchet Progressive Type II Censored Data with Binomial Removals
}

\author{
Mohamed Mubarak ${ }^{1,2}$ \\ ${ }^{1}$ Mathematics Department, Faculty of Science, Minia University, El-Minia 61519, Egypt \\ ${ }^{2}$ Mathematics Department, University College in Lieth, Umm Al-Qura University, Makkah 311, Saudi Arabia
}

Correspondence should be addressed to Mohamed Mubarak, mubarak_7061111@yahoo.com

Received 17 March 2011; Revised 16 June 2011; Accepted 17 June 2011

Academic Editor: Chun-Ping Lin

Copyright ( 2012 Mohamed Mubarak. This is an open access article distributed under the Creative Commons Attribution License, which permits unrestricted use, distribution, and reproduction in any medium, provided the original work is properly cited.

This paper considers the estimation problem for the Frèchet distribution under progressive Type II censoring with random removals, where the number of units removed at each failure time has a binomial distribution. We use the maximum likelihood method to obtain the estimators of parameters and derive the sampling distributions of the estimators, and we also construct the confidence intervals for the parameters and percentile of the failure time distribution.

\section{Introduction}

Recently, the extreme value distribution is becoming increasingly important in engineering statistics as a suitable model to represent phenomena with usually large maximum observations. In engineering circles, this distribution is often called the Frèchet model. It is one of the pioneers of extreme value statistics. The Frèchet (extreme value type II) distribution is one of the probability distributions used to model extreme events. The generalization of the standard Frèchet distribution has been introduced by Nadarajah and Kotz [1] and Abd-Elfattah and omima [2]. There are over fifty applications ranging from accelerated life testing through to earthquakes, floods, rain fall, queues in supermarkets, sea currents, wind speeds, and track race records, see Kotz and Nadarajah [3]. Censoring arises in a life test when exact lifetimes are known for only a portion of test units and the remainder of the lifetimes are known only to exceed certain values under an experiment. There are several types of censored test. One of the most common censoring schemes is Type II censoring. In a Type II censoring, a total of $n$ units is placed on test, but instead of continuing until all $n$ units have failed, the test is terminated at the time of the $m$ th $(1 \leq m \leq n)$ unit failure. Type II censoring with different failure time distribution has been studied by many authors including Mann et al. [4], Lawless [5], and Meeker and Escobar [6]. If an experiment desires to remove live units at points other than the final termination point of the life test, the above described scheme will not be of use to experimenter. Type II censoring does not allow for units to be lost or removed from the test at points other than the final termination point see Balakrishnan and Aggarwala [7, Chapter 1].

A generalization of Type II censoring is progressive Type II censoring. Under this scheme, $n$ units are placed on test at time zero, and $m$ failure are going to be observed. When the first failure is observed, $r_{1}$ of surviving units are randomly selected, removed, and so on. This experiment terminates at the time when the $m$ th failures is observed and remaining $r_{m}=n-r_{1}-r_{2}-\cdots-r_{m-1}-m$ surviving units are all removed. The statistical inference on the parameters of failure time distribution under progressive Type II censoring has been studied by several authors [7-10]. Note that, in this scheme, $r_{1}, \ldots, r_{m}$ are all prefixed. However, in some practical situations, these numbers may occur at random [11]. In some reliability experiments, an experimenter may decide that it is inappropriate or too dangerous to carry on the testing on some of the tested units even though 
these units have not failed. In these cases, the pattern of removal at each failure is random. We assume that, any test unit being dropped out from the life test is independent of the others but with the same probability $p$. Then Tea et al. [12] indicated that the number of test units removed at each failure time has a binomial distribution.

In this paper, we will make inference on the parameters of three-parameter Frèchet distribution under progressive type II censoring with binomial removals. The maximum likelihood estimators (MLEs) for the parameters in an explicit and implicit form are obtained in Section 3. Section 4 discusses the sampling distribution of the MLEs and constructs the confidence intervals for the parameters. The estimator and confidence interval for the percentile of failure time distribution are also presented in Section 4.

\section{Description of the Model}

Let random variable $X$ has a three-parameter Frèchet distribution. The probability density function and cumulative distribution function are

$$
\begin{aligned}
& f(x)=\left(\frac{\alpha}{\beta}\right)\left(\frac{\beta}{x-\theta}\right)^{\alpha+1} \exp \left[-\left(\frac{\beta}{x-\theta}\right)^{\alpha}\right], \\
& (x>\theta, \alpha, \beta>0), \\
& F(x)=\exp \left[-\left(\frac{\beta}{x-\theta}\right)^{\alpha}\right], \quad(x>\theta, \alpha, \beta>0),
\end{aligned}
$$

respectively, where $\alpha$ is continuous shape parameter, $\beta$ is continuous scale parameter, and $\theta$ is continuous location parameter $(\theta=0$ yields the two-parameter Frèchet distribution).

\section{Estimation of Parameters}

Suppose $n$ independent units are placed on a test with the corresponding life times being identically distributed with probability density function $f_{X}(x)$ and cumulative distribution function $F_{X}(x)$. For simplicity of notation, let $X_{1}, X_{2}, \ldots, X_{m}$ denote a progressively Type II censored sample. Then, the joint probability density function of all $m$ progressively Type II-censored order statistics is

$$
\begin{aligned}
& f_{X_{1}, \ldots, X_{m}}\left(x_{1}, \ldots, x_{m}\right) \\
& \quad=k \prod_{i=1}^{m} f_{X}\left(x_{i}\right)\left[1-F_{X}\left(x_{i}\right)\right]^{r_{i}}, \quad x_{1}<\cdots<x_{m}
\end{aligned}
$$

where $k=n\left(n-r_{1}-1\right) \cdots\left(n-r_{1}-\cdots-r_{m-1}-m+1\right)$. Thus, for a progressive Type II with predetermined number of removals $R=r$, the conditional likelihood and Loglikelihood functions, respectively, can be written as

$$
\begin{aligned}
& L(\alpha, \beta, \theta \mid R=r) \\
& =\frac{k \alpha^{m}}{\beta^{m}} \prod_{i=1}^{m}\left[\frac{\beta}{x_{i}-\theta}\right]^{(\alpha+1)} \\
& \quad \times\left[1-\exp \left(-\left[\frac{\beta}{x_{i}-\theta}\right]^{\alpha}\right)\right]^{r_{i}} \exp \left(-\left[\frac{\beta}{x_{i}-\theta}\right]^{\alpha}\right), \\
& L^{*}(\alpha, \beta, \theta \mid R=r) \\
& =\ln k+m \ln \alpha+m \alpha \ln \beta-(\alpha+1) \sum_{i=1}^{m} \ln \left(x_{i}-\theta\right) \\
& -\beta^{\alpha} \sum_{i=1}^{m}\left(x_{i}-\theta\right)^{-\alpha}+\sum_{i=1}^{m} r_{i} \ln \left[1-\exp \left(-\left[\frac{\beta}{x_{i}-\theta}\right]^{\alpha}\right)\right],
\end{aligned}
$$

where $k$ is defined in (3). Now, suppose that an individual unit being removed from the life test is independent of the others but with the same probability $p$. Then, the number of units removed at each failure time follows a binomial distribution such that

$$
P\left(R_{1}=r_{1}\right)=\left(\begin{array}{c}
n-m \\
r_{1}
\end{array}\right) p^{r_{i}}(1-p)^{n-m-r_{1}}
$$

where $0 \leq r_{1} \leq n$

$$
\begin{aligned}
P\left(R_{i}=\right. & \left.r_{i} \mid R_{i-1}=r_{i-1}, \ldots, R_{1}=r_{1}\right) \\
= & \left(\begin{array}{c}
n-m-\sum_{k=1}^{i-1} r_{k} \\
r_{i}
\end{array}\right) \times p^{r_{i}}(1-p)^{n-m-\sum_{k=1}^{i-1} r_{k}},
\end{aligned}
$$

where $0 \leq r_{i} \leq n-m-\sum_{k=1}^{i} r_{k}, i=2, \ldots, m-1$. Suppose further that $R_{i}$ is independent of $X_{i}$. Then, the Log-likelihood function can be expressed as

$$
L^{*}(\alpha, \beta, \theta)=L^{*}(\alpha, \beta, \theta \mid R=r) P(R=r),
$$

where

$$
\begin{aligned}
& P(R=r) \\
& =P\left(R_{m-1}=r_{m-1} \mid R_{m-2}=r_{m-2}, \ldots, R_{1}=r_{1}\right) \\
& \cdots P\left(R_{2}=r_{2} \mid R_{1}=r_{1}\right) P\left(R_{1}=r_{1}\right) \text {, } \\
& P(R=r) \\
& =\frac{(n-m) !}{\prod_{i=1}^{m-1} r_{i} !\left(n-m-\sum_{i=1}^{m-1} r_{i}\right) !} \\
& \times p^{\sum_{i=1}^{m-1} r_{i}}(1-p)^{(m-1)(n-m)-\sum_{i=1}^{m-1}(m-i) r_{i}} .
\end{aligned}
$$


Independently, the MLE of parameter $p$ can be obtained by maximizing (10). Thus, we find immediately

$$
\hat{p}=\frac{\sum_{i=1}^{m-1} r_{i}}{(m-1)(n-m)-\sum_{i=1}^{m-1}(m-i-1) r_{i}} .
$$

Note that $P(R=r)$ does not depend on the parameters $\alpha, \beta$, and $\theta$, and hence the MLE's of the parameters can be derived from (5) by differentiating with respect to $\alpha, \beta$, and $\theta$ and equating to zero, in this case we have

$$
\begin{aligned}
& m-\sum_{i=1}^{m}\left[\frac{\beta}{x_{i}-\theta}\right]^{\alpha} \\
& +\sum_{i=1}^{m} \frac{r_{i}\left[\beta /\left(x_{i}-\theta\right)\right]^{\alpha} \exp \left(-\left[\beta /\left(x_{i}-\theta\right)\right]^{\alpha}\right)}{1-\exp \left(-\left[\beta /\left(x_{i}-\theta\right)\right]^{\alpha}\right)}=0 \\
& \sum_{i=1}^{m}\left(\frac{1+\alpha}{\alpha}\left[\frac{\beta}{x_{i}-\theta}\right]-\left[\frac{\beta}{x_{i}-\theta}\right]^{(\alpha+1)}\right. \\
& \left.+\frac{r_{i}\left[\beta /\left(x_{i}-\theta\right)\right]^{(\alpha+1)} \exp \left(-\left[\beta /\left(x_{i}-\theta\right)\right]^{\alpha}\right)}{1-\exp \left(-\left[\beta /\left(x_{i}-\theta\right)\right]^{\alpha}\right)}\right)=0, \\
& \frac{m}{\alpha}+m \ln \beta \\
& \begin{aligned}
+\sum_{i=1}^{m}( & -\left[\frac{\beta}{x_{i}-\theta}\right]^{\alpha} \ln \left[\frac{\beta}{x_{i}-\theta}\right] \\
+ & \left.\frac{r_{i} \ln \left[\beta /\left(x_{i}-\theta\right)\right] \exp \left(-\left[\beta /\left(x_{i}-\theta\right)\right]^{\alpha}\right)}{1-\exp \left(-\left[\beta /\left(x_{i}-\theta\right)\right]^{\alpha}\right)}\right)=0,
\end{aligned}
\end{aligned}
$$

since (12) cannot be solved analytically for estimators $\hat{\alpha}, \hat{\beta}$, and $\hat{\theta}$. Hence, in this paper, the numerical solution is used to solve the problem.

\section{Some Further Results}

In this section, we are going to derive the sampling distributions of the MLE's and obtain the confidence intervals for the parameters [13-15]. In addition, we will obtain the MLE and confidence interval for the percentile of failure time distribution.

4.1. Distributions and Confidence Intervals for the Parameters. Let $X_{1}<X_{2}<\cdots<X_{m-1}<X_{m}$ denote a progressively Type II censored sample from a three-parameter Frèchet distribution with censored scheme $R=\left(r_{1}, \ldots, r_{m}\right)$. Let $Y_{i}=$ $\left[\left(x_{i}-\theta\right) / \beta\right]^{-\alpha}, i=1, \ldots, m$. It can be seen that, $Z_{1}<Z_{2}<$ $\cdots<Z_{m}$ is a progressively Type II censored sample from an exponential distribution with mean 1 . Let us consider the following transformation

$$
\begin{aligned}
Z_{1} & =n Y_{1} \\
Z_{2} & =\left(n-r_{1}-1\right)\left(Y_{2}-Y_{1}\right) \\
\vdots & \vdots \\
Z_{m} & =\left(n-r_{1}-\cdots-r_{m-1}-m+1\right)\left(Y_{m}-Y_{m-1}\right) .
\end{aligned}
$$

Thomas and Wilson [16] showed that the generalized spacings $Z_{1}, Z_{2}, \ldots, Z_{m}$ as defined in the previous equation (13) are all independent and identically distributed as standard exponential. $V=2 Z_{1}=2 n\left[\left(x_{i}-\theta\right) / \beta\right]^{-\alpha}$ has a Chi-square distribution with 2 degrees of freedom. We can also write the numerator of $\hat{\beta}$ as the sum of $m-1$ independent generalized spacings, that is, $2 \sum_{i=1}^{m}\left(r_{i}+1\right) Y_{i}-n Y_{1}=2 \sum_{i=2}^{m} Z_{i}$. Therefore, we can find that, conditionally on a fixed set of $R=$ $\left(r_{1}, \ldots, r_{m}\right), U=2 \sum_{i=1}^{m}\left(r_{i}+1\right) Y_{i}-n Y_{1}$ has a Chi-square distribution with $2 m-2$ degrees of freedom. It is also easily seen that $V$ and $U$ are independent. Let

$$
\begin{aligned}
& T_{1}=\frac{U}{(m-1) V}=\frac{\sum_{i=1}^{m}\left(r_{i}+1\right) Y_{i}-n Y_{1}}{n(m-1) Y_{1}}, \\
& T_{2}=U+V=2 \sum_{i=1}^{m}\left(r_{i}+1\right) Y_{i} .
\end{aligned}
$$

It is easy to show that, $T_{1}$ has an $F$ distribution with $2 m-2$ and 2 degrees of freedom, and $T_{2}$ has a Chi-square distribution with $2 m$ degrees of freedom. Furthermore, $T_{1}$ and $T_{2}$ are independent.

Theorem 1. Suppose that $w_{1}<w_{2}<\cdots<w_{m}$. Let

$$
T_{1}(w)=\frac{\sum_{i=1}^{m}\left(r_{i}+1\right)\left(w_{i}-\theta\right)^{-\alpha}-n\left(w_{1}-\theta\right)^{-\alpha}}{n(m-1)\left(w_{1}-\theta\right)^{-\alpha}} .
$$

Then, $T_{1}(w)$ is strictly increasing in $w$ for any $w>0$, furthermore, if $t>0$, the equation $T_{1}(w)=t$ has unique solution for any $w>0$.

Suppose that, $X_{i}, i=1, \ldots, m$ are order statistics of a progressively Type II censored sample of size $n$ from the Frèchet distribution, with censoring scheme $\left(r_{1}, \ldots, r_{m}\right)$ the a $100(1-\phi)$ confidence interval for $\alpha$ is

$$
\begin{aligned}
& \Psi\left(X_{1}, \ldots, X_{m}, F_{\phi / 2}(2 m-2,2)\right) \\
& \quad<\alpha<\Psi\left(X_{1}, \ldots, X_{m}, F_{1-\phi / 2}(2 m-2,2)\right) .
\end{aligned}
$$


Such that,

$1-\phi$

$$
\begin{aligned}
=P[ & \left.F_{\phi / 2}(2 m-2,2)<T_{1}<F_{1-\phi / 2}(2 m-2,2)\right] \\
=P[ & F_{\phi / 2}(2 m-2,2) \\
& <\frac{\sum_{i=1}^{m}\left(r_{i}+1\right)\left(x_{i}-\theta\right)^{-\alpha}-n\left(x_{1}-\theta\right)^{-\alpha}}{n(m-1)\left(x_{1}-\theta\right)^{-\alpha}} \\
& \left.<F_{1-\phi / 2}(2 m-2,2)\right] .
\end{aligned}
$$

Furthermore, the $100(1-\phi)$ joint confidence region for $\alpha$ and $\beta$ is determined by the following inequalities:

$$
\begin{aligned}
& \Psi\left(X_{1}, \ldots, X_{m}, F_{(1+\sqrt{1-\phi}) / 2}(2 m-2,2)\right), \\
& <\alpha<\Psi\left(X_{1}, \ldots, X_{m}, F_{(1-\sqrt{1-\phi}) / 2}(2 m-2,2)\right) \\
& \frac{\chi_{(1+\sqrt{1+\phi}) / 2}^{2}(2 m)}{\left[2 \sum_{i=1}^{m}\left(r_{i}+1\right)\left(x_{i}-\theta\right)^{\alpha}\right]^{1 / \alpha}}<\beta<\frac{\chi_{(1+\sqrt{1-\phi}) / 2}^{2}(2 m)}{\left[2 \sum_{i=1}^{m}\left(r_{i}+1\right)\left(x_{i}-\theta\right)^{\alpha}\right]^{1 / \alpha}} .
\end{aligned}
$$

Such that,

$$
\begin{aligned}
& 1-\phi=\sqrt{1-\phi} \sqrt{1-\phi} \\
& =\left[P F_{(1+\sqrt{1-\phi}) / 2}(2 m-2,2)<T_{1}\right. \\
& \left.\quad<F_{(1-\sqrt{1-\phi}) / 2}(2 m-2,2)\right] \\
& \quad \times P\left[\chi_{(1+\sqrt{1-\phi}) / 2}^{2}(2 m)<T_{2}<\chi_{(1-\sqrt{1-\phi}) / 2}^{2}(2 m)\right] .
\end{aligned}
$$

4.2. Confidence Interval of $x_{F}$. In reliability analysis [17, 18 ], we are not only interested in making inference about parameter but also interested in deriving inference about percentiles of the failure time distribution. Let $x_{F}$ be the $100_{F}$ th percentile of the failure time distribution. One can obtain $x_{F}$ by solving $F_{X}\left(x_{F}\right)=p$ where $F_{X}(\cdot)$ is as given in (2). Then it easy to see that $x_{F}=\theta-\beta(\log p)^{-1 / \alpha}$. Consequently, the MLE of $x_{F}$ is given by $x_{F}=\hat{\theta}-$ $\hat{\beta}(\log p)^{-1 / \hat{\alpha}}$. Confidence interval for $x_{F}$ can be derived by using the pivotal quantity $U_{F}=\left[\left(x_{F}-\hat{\theta}\right) / \hat{\beta}\right]^{-\hat{\alpha}}$ if $\left(u_{f, 1-\phi}\right)^{-\alpha}$ is the $(1-\phi)$ th percentile of $U_{F}$, then

$$
1-\phi=P\left[U_{F} \leq u_{F, 1-\phi}\right]=P\left[x_{F} \geq \hat{\theta}-\hat{\beta} u_{F, 1-\phi}\right] .
$$

Hence, $\hat{\theta}-\hat{\beta} u_{F, 1-\phi}$ is a lower confidence limit for $x_{F}$ with confidence coefficient $1-\phi$. We can rewrite (21) as

$$
1-\phi=P\left[\frac{V}{2 n}+\lambda U \leq-\ln p\right]
$$

such that, $V \sim \chi^{2}(2), U \sim \chi^{2}(2 m-2)$, and $\lambda=-u_{F, 1-\phi} / 2 m$. In addition, $V$ and $U$ are independent, which is discussed in the previous subsection. Hence, we need to find the value of $\lambda$ by using solving (22). There are several methods for computing $\lambda$, see Engelhardt and Bain [19].

\section{Conclusions}

We develop some results on a three-parameter Frèchet distribution when progressive Type II censoring with binomial removals is performed. We derive the MLEs and confidence for the parameters. The MLE and confidence interval for the percentiles of failure time distribution are obtained. In practice, it is often useful to have an idea of the duration of a life test. Therefore, it is important to compute the expected time required to complete a life test. In the case of progressively type II-censored sampling plan with binomial removals, one can obtain this information by calculating the expectation of the $m$ th order statistic. In fact, we believe that the value of removal probability $p$ is very important when we compare the expected test times of progressive Type II censoring with binomial removals and complete sampling plan. In addition, the removal probability $p$ may not be fixed for each stage. Such belief is not discussed in this paper and we will be investigated in the future.

\section{List of Symbols}

$\begin{array}{ll}f(x): & \text { Probability density function } \\ F(x): & \text { Cumulative distribution function } \\ \alpha, \beta, \theta: & \text { Three-parameter Frèchet distribution } \\ R: & \text { Number of removals } \\ p: & \text { Removal probability } \\ \chi^{2}: & \text { Chi-square } \\ F: & \text { F-distribution } \\ 100(1-\phi): & \text { Confidence interval } \\ X_{F}: & \text { 100-th percentile of the failure time } \\ & \text { distribution. }\end{array}$

\section{References}

[1] S. Nadarajah and S. Kotz, "The Exponentiated Frechet Distribution,” Interstat Electronic Journal, 2003, http://interstat .statjournals.net/YEAR/2003/articles/.

[2] A. M. Abd-Elfattah and A. M. Omima, "Estimation of the unknown parameters of the generalized Frechet distribution," Journal of Applied Sciences Research, vol. 5, no. 10, pp. 13981408, 2009.

[3] S. Kotz and S. Nadarajah, Extreme Value Distributions: Theory and Applications, Imperial College Press, London, UK, 2000.

[4] N. R. Mann, R. E. Schafer, and N. D. Singpurwalla, Methods for Statistical Analysis of Reliability and Life Data, John Wiley \& Sons, New York, NY, USA, 1974.

[5] J. F. Lawless, "Statistical Methods and Methods for Lifetime Data, John Wiley \& Sons, New York, NY, USA, 1982.

[6] W. Q. Meeker and L. A. Escobar, Statistical Methods for Reliability Data, John Wiley \& Sons, New York, NY, USA, 1998.

[7] N. Balakrishnan and R. Aggarwala, Progressive CensoringTheory, Moethods and Applications, Birkhäauser, Boston, Mass, USA, 2000.

[8] A. C. Cohen, "Progressively censored samples in life testing," Technometrics, vol. 5, pp. 327-339, 1976.

[9] N. R. Mann, "Best liner invariant estimation for Weibull parameters under progressive censoring," Technometrics, vol. 13, pp. 521-533, 1971. 
[10] R. Viveros and N. Balakrishnan, "Interval estemation of parameters of life from progressivve censoring data," Technometrics, vol. 36, pp. 84-91, 1994.

[11] H. K. Yuen and S. K. Tse, "Parameters estimation for weibull distributed lifetimes under progressive censoring with random removals," Journal of Statistical Computation and Simulation, vol. 55, no. 1-2, pp. 57-71, 1996.

[12] S. K. Tae, C. Yang, and H. K. Yuen, "Statistical analysis of Weibull distributed lifetime data under Type II prograssive censoring with binomial removals," Journal of Applied Statistics, vol. 27, pp. 1033-1043, 2000.

[13] K. Alakuş, "Confidence intervals estimation for survival function in weibull proportional hazards regression based on censored survival time data," Scientific Research and Essays, vol. 5, no. 13, pp. 1589-1594, 2010.

[14] M. Maswadah, "Conditional confidence interval estimation for the inverse weibull distribution based on censored generalized order statistics," Journal of Statistical Computation and Simulation, vol. 73, no. 12, pp. 887-898, 2003.

[15] J. A. Griggs and Y. Zhang, "Determining the confidence intervals of Weibull parameters estimated using a more precise probability estimator," Journal of Materials Science Letters, vol. 22, no. 24, pp. 1771-1773, 2003.

[16] D. R. Thomas and W. M. Wilson, "Linear order statistic estimation for the two parameter Weibull and extreme value distribution from type -II progressively censored samples," Technometrics, vol. 14, pp. 679-691, 1972.

[17] M. Han, "Estimation of failure probability and its applications in lifetime data analysis," International Journal of Quality, Statistics, and Reliability, vol. 2011, Article ID 719534, 6 pages, 2011.

[18] S. Loehnert, "About statistical analysis of qualitative survey data," International Journal of Quality, Statistics, and Reliability, vol. 2010, Article ID 849043, 12 pages, 2010.

[19] M. Engelhardt and L. J. Bain, "Tolerance limits and confidence limits on reliability for the two parameter exponential distribution," Technometrics, vol. 20, pp. 37-39, 1978. 

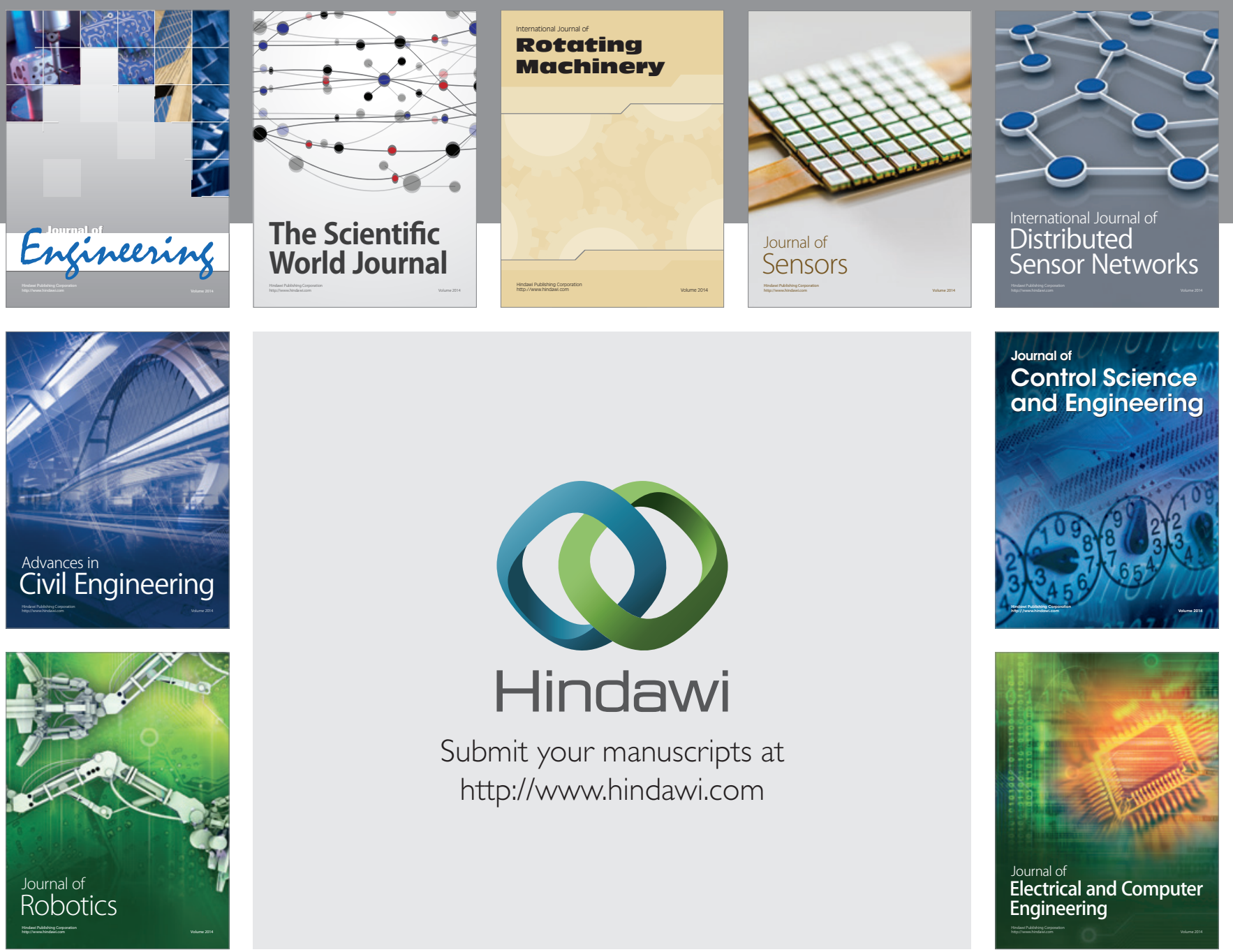

Submit your manuscripts at

http://www.hindawi.com
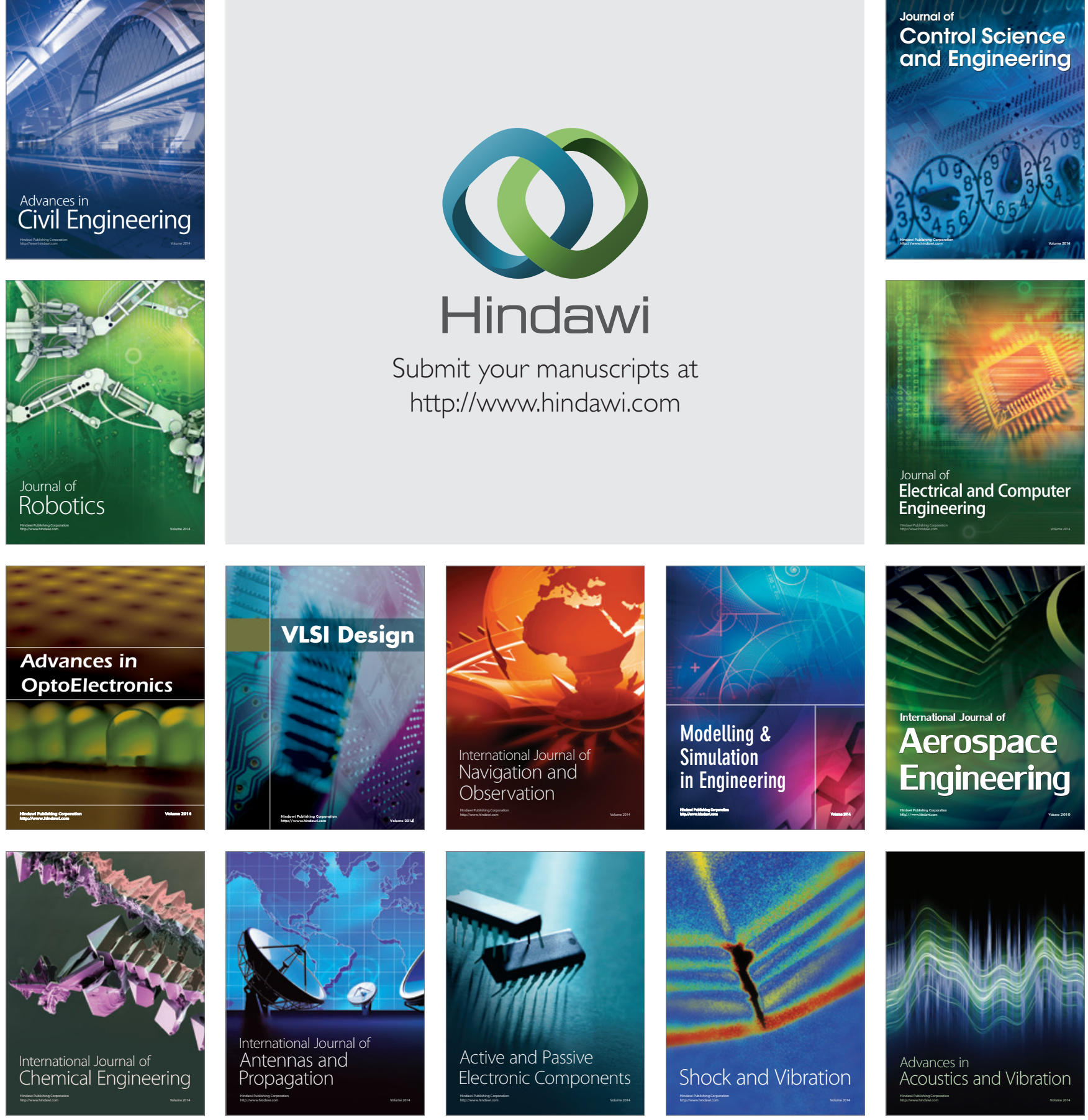